\title{
Homograft aortic root replacement with modified Cabrol extension using cryopreserved femoral artery for bioprosthetic aortic valve endocarditis
}

\author{
Timothy M. Guenther, MD, ${ }^{\mathrm{a}, \mathrm{b}}$ Luis Godoy, MD, ${ }^{\mathrm{a}}$ Sarah A. Chen, MD, ${ }^{\mathrm{a}}$ and Victor M. Rodriguez, MD,
} Sacramento and Travis AFB, Calif

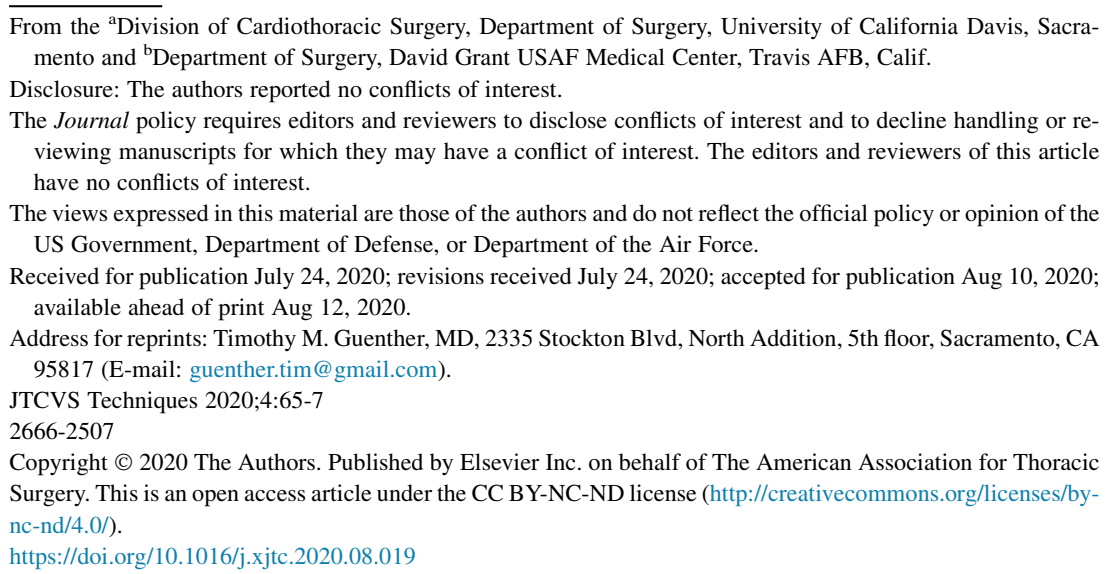
Video clip is available online.

Prosthetic valve endocarditis (PVE) is a serious complication after aortic valve replacement, with a mortality rate between $18 \%$ and $30 \%{ }^{1}$ Although some patients can be effectively managed with prolonged courses of antibiotics, surgical indications exist and include abscess formation, development of heart block or failure, persistent bacteremia, and infection with certain organisms, such as fungi and certain virulent bacteria. ${ }^{2}$ Operative intervention entails resection of infected/necrotic material, drainage of associated abscesses, and commonly aortic root reconstruction. We report a patient with PVE associated with significant periannular abscess treated with a homograft aortic root replacement with coronary reimplantation using a modified Cabrol extension via a cryopreserved femoral artery.

\section{CASE DESCRIPTION}

A 59-year-old man with history of atrial fibrillation and streptococcal native aortic valve endocarditis who had undergone a $29-\mathrm{mm}$ bioprosthetic aortic valve replacement 3 years earlier presented with fever and left lower extremity injury after a fall. Given a concern for compartment syndrome, a left lower extremity fasciotomy was performed. Postoperatively, the patient had persistent fever and

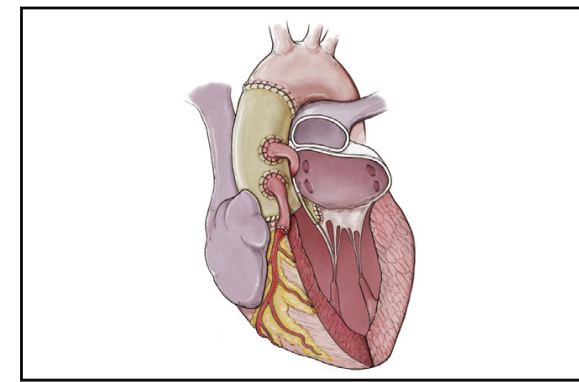

Homograft aortic root replacement with biologic modified Cabrol Extension.

CENTRAL MESSAGE

We describe a patient with extensive prosthetic valve endocarditis treated with a homograft aortic root replacement and a modified Cabrol extension via cryopreserved femoral artery.

See Commentaries on pages 68 and 70.

leukocytosis, with blood cultures growing Staphylococcus lugdunensis. Serial echocardiograms showed rapidly progressing endocarditis with bioprosthetic valve leaflet thickening, vegetation formation, and periannular abscess formation (Video 1). He also developed acute mitral valve insufficiency and new right bundle branch block. Crosssectional imaging confirmed progressive thickening around the aortic root concerning for a large aortic root abscess and new outpouchings at the sinuses of Valsalva suggestive of pseudoaneurysm formation (Figure 1).

In the operating room, cardiac arrest was achieved using antegrade cardioplegia. The bioprosthetic aortic valve was severely infected and nearly entirely dehisced. The annular abscess was circumferential with associated gross purulence. The aortic-mitral continuity was eroded, and the anterior leaflet of the mitral valve was partially detached.

The operation involved extensive root debridement, reestablishment of aortic-mitral continuity with a bovine pericardial patch, suture resuspension of the anterior leaflet of the mitral valve, and a $25-\mathrm{mm}$ homograft root replacement 


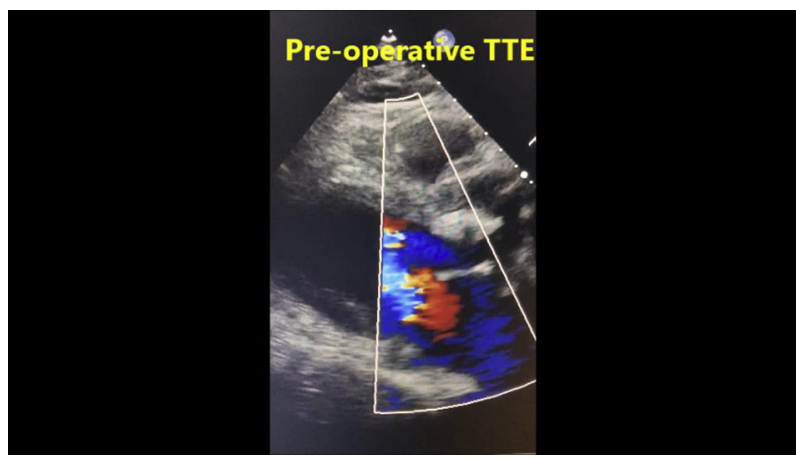

VIDEO 1. Video summary of the case report including clips of preoperative, operative, and postoperative echocardiograms. (0:14) Preoperative transthoracic echocardiogram (parasternal long-axis view) showing thickened prosthetic valve leaflets and vegetations. (0:19) Preoperative transthoracic echocardiogram (parasternal long-axis view) showing thickened prosthetic valve leaflets and vegetations with color flow. (0:31) Intraoperative transesophageal echocardiogram demonstrating large root abscess. (0:38) Intraoperative transesophageal echocardiogram demonstrating large root abscess with color flow. (0:50) Postoperative transthoracic echocardiogram (parasternal long-axis view) showing the aortic root homograft replacement. (0:55) Postoperative transthoracic echocardiogram (parasternal long-axis view) showing the aortic root homograft replacement with color flow. Video available at: https://www.jtcvs.org/article/S2666-2507(20) 30394-1/fulltext.

(Figure 2). Because of extensive aortic root scar tissue and inflammation, both coronaries were immobile and not amenable to direct aortic reimplantation. Use of a saphenous vein conduit was considered but was deemed infeasible owing to poor vessel quality bilaterally (associated thrombophlebitis from recent surgery on the left and small

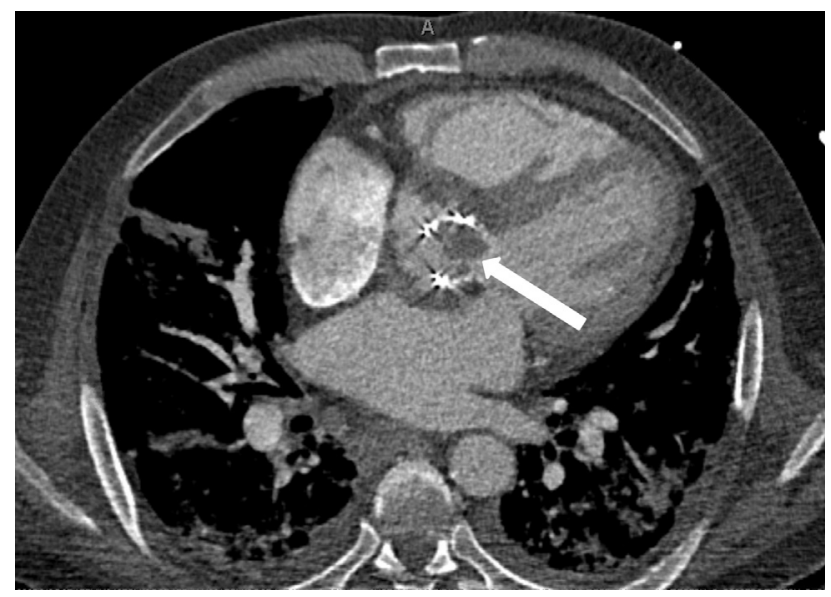

FIGURE 1. Preoperative computed tomography scan in a patient with bioprosthetic valve endocarditis showing thickening of the bioprosthetic aortic valve leaflets ( $29 \mathrm{~mm}$ annulus), valvular vegetations (highlighted by white arrow), and thickening of the surrounding soft tissue around the aortic root concerning for large aortic root abscess. size on the right). In the setting of gross infection, a modified Cabrol extension to both coronary ostia using a 9-mm cryopreserved superficial femoral artery homograft was performed.

The patient's heart block resolved postoperatively, and computed tomography angiography on postoperative day 10 confirmed coronary patency. The patient's postoperative course was complicated by respiratory failure necessitating tracheostomy and malnutrition requiring gastrostomy tube placement. He was discharged to a rehabilitation facility on postoperative day 94 and has since been discharged home. He remains on life-long oral doxycycline.

Verbal consent was obtained from the patient for publication of this case report.

\section{DISCUSSION}

Given the morbidity and mortality associated with PVE, consideration of the optimal surgical technique is essential. The use of prosthetic material in infected fields is generally avoided whenever possible, to prevent recurrence of infection. Randomized prospective studies comparing homograft and prosthetic material for aortic root replacement in PVE are lacking; a retrospective observational review of 29 patients showed no difference in complication rate or overall mortality. ${ }^{3}$ However, in the setting of extensive infection in PVE, homograft aortic root replacement represents a viable method of repair, with reported reinfection rates of $<5 \%$.

Establishment of coronary blood flow after root replacement in PVE can be challenging given scarring from the original aortic valve replacement and associated inflammatory changes near the root/coronary ostia from infection. The use of prosthetic and saphenous vein conduits has been described in the setting of immobile coronary arteries not amenable to direct aortic reimplantation. Our patient had no viable saphenous vein, and given the associated extensive root abscess, we opted for a biological conduit over prosthetics. A superficial femoral artery homograft was found to be an appropriately-sized conduit to allow aortic reimplantation of the frozen native coronary arteries without tension using a modified Cabrol extension. The original Cabrol technique described anastomosing an 8$\mathrm{mm}$ Dacron graft to both coronary arteries in an end-toend fashion and then anastomosing the midportion of this graft onto the aorta in a side-to-side fashion. ${ }^{5}$ However, modifications of this technique have been described, including direct implantation of separate grafts from each coronary artery onto the aorta, as was the technique used in the present case.

In conclusion, this case highlights a unique method of repair in the setting of aortic root replacement for PVE complicated by extensive root abscess and immobile coronary arteries. 


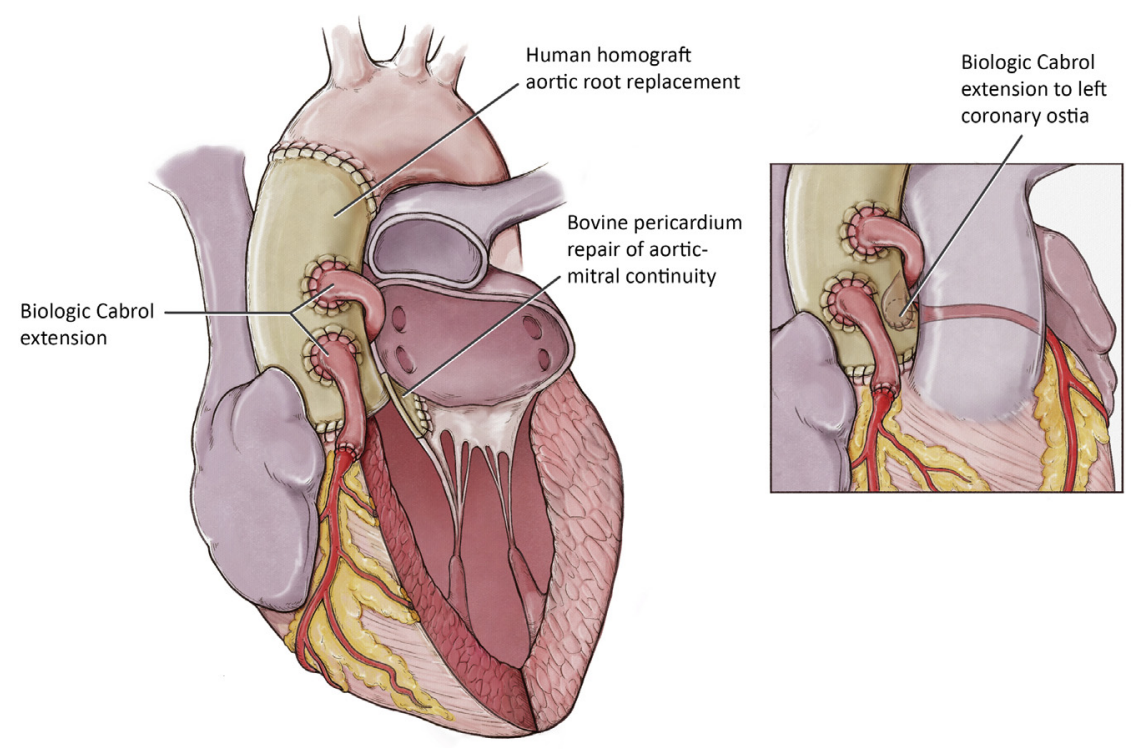

FIGURE 2. Illustration of operative repair in a patient with bioprosthetic valve endocarditis with root abscess. Given the extensive infection, a homograft root replacement was performed after aortic-mitral continuity was reestablished with a bovine pericardial patch repair and the anterior leaflet of the mitral valve was resuspended. Direct reimplantation of the coronary arteries was not possible, and with no available saphenous vein conduit and in the setting of extensive infection, the "immobile" coronary arteries were reimplanted using a modified Cabrol extension with homograft femoral artery. A mildly aneurysmal native ascending aorta allowed for a fairly anterior placement of the left coronary artery without kinking or concern for compression between the neoaorta and pulmonary artery.

\section{References}

1. Wang A, Athan E, Pappas PA, Fowler VG Jr, Olaison L, Paré C, et al. Contemporary clinical profile and outcome of prosthetic valve endocarditis. JAMA. 2007; 297:1354-61.

2. Baddour LM, Wilson WR, Bayer AS, Fowler VG Jr, Tleyjeh IM, Rybak MJ, et al. Infective endocarditis in adults: diagnosis, antimicrobial therapy, and management of complications: a scientific statement for healthcare professionals from the American Heart Association. Circulation. 2015;132:1435-86.

3. Leyh RG, Knobloch K, Hagl C, Ruhparwar A, Fischer S, Kofidis T, et al. Replacement of the aortic root for acute prosthetic valve endocarditis: prosthetic compos- ite versus aortic allograft root replacement. J Thorac Cardiovasc Surg. 2004;127: 1416-20.

4. Musci M, Weng Y, Hübler M, Amiri A, Pasic M, Kosky S, et al. Homograft aortic root replacement in native or prosthetic active infective endocarditis: twenty-year single-center experience. J Thorac Cardiovasc Surg. 2010;139: 665-73.

5. Cabrol C, Pavie A, Gandjbakhch I, Villemot JP, Guiraudon G, Laughlin L, et al Complete replacement of the ascending aorta with reimplantation of the coronary arteries: new surgical approach. J Thorac Cardiovasc Surg. 1981;81: $309-15$. 